\title{
Study on the flexural behavior of poplar beams externally strengthened by BFRP strips
}

\author{
Qing Liu, Shengqiang Ma* (i) and Xia Han
}

\begin{abstract}
Poplar plants with peculiar characteristics grow extensively and naturally in the Northern Hemisphere, and such poplar is the main timber material in Xinjiang, China. However, poplar has defects that affect its ultimate bearing capacity. This study investigates the flexural behavior of the timber members from poplar. Three lengths of basalt fiberreinforced polymer (BFRP) strips were used to strengthen timber beams from poplar with rectangular cross-sections. From the experimental data, the timber beams strengthened by 1900-mm-long and 50-mm-wide BFRP strips showed the best ultimate bearing capacity and maximum deflection of mid-span compared with the other two strengthened beams. The increments were $77.8 \%$ and $110.1 \%$ compared with the control beam. The assumption "plane section remains plane" remains practicable in the poplar beams bonded by BFRP strips, and the neutral axis of the strengthened beams had a tendency to move toward the tensile zone. Furthermore, the processes of the flexural behavior of the specimens were simulated by finite element analysis modeling, but all the analyzed values of ultimate bearing capacity and deflection of the beams are higher than those of the experiment. These values can roughly be used as reference for experimental results. However, accurate modeling should be developed and modified in subsequent research on timber beams from Xinjiang poplar.
\end{abstract}

Keywords: Flexural behavior, Poplar, Timber beam, BFRP strip, Strengthening, FEA

\section{Introduction}

The application of wooden structures has a thousandyear history in China. Many wooden structures, such as palaces, dwellings, and temples, which were built in different historical periods are found in China, Japan, Korea, and Southeast Asian countries [1]. In recent years, wooden structures have become attractive and popular because of their excellent characteristics, such as being lightweight, comfortable, and environmentally friendly. Poplar (genus Populus), as an alternative building material, has unique features, such as energy efficiency, good formability, good thermal insulation and sound insulation, fast growth and high productivity, and low cost; hence, poplar has been widely grown in the Northern Hemisphere [2]. Poplar timber has been gradually used in wooden structures as bearing bending loads. Although

*Correspondence: masq@xju.edu.cn

School of Architectural and Civil Engineering, Xinjiang University, Xinjiang 830047, China wooden structures are important building forms, timber has natural defects, such as fine cracks, knots, and crossgrain that affect bearing capacity. All these features of wood result in decreases in the capacity of wooden structures, especially wood elements such as beams. Therefore, finding an effective strengthening method to retrofit wooden structures for improving capacity is necessary. Such a method is of great significance for ancient and modern wooden buildings.

Traditional strengthening techniques for timber structural members normally apply steel and aluminum plates, or simply timber patches [3-5]. Nevertheless, these strengthening methods may impose additional dead load on a structure, and the costs of transportation and installation may be increased accordingly. Furthermore, due to thermal loads applied on timber structures, steel parts and fittings are susceptible to corrosion and aluminum plates may deform easily [6]. Therefore, fiberreinforced polymer (FRP) is an alternative strengthening material used to increase the bearing capacity of timber 
structures, especially when load-carrying ability and stiffness of timber are required.

Improving the flexural capacity of timber beams with externally epoxy-bonded FRP strips on the tensile zone is not a new technique. Several research and studies have been conducted on the application of carbon (CFRP), glass (GFRP), and aramid (AFRP), which are used to strengthen timber members or concrete structures [7-9]. For timber specimens subjected to bending moment, remarkable failure occurs frequently at the lower beam side because of tensile stress. FRP with excellent mechanical properties is bonded at the bottom of a timber beam, increasing tensile strength and stiffness. In pioneering studies [7], timber beams wrapped in GFRP were studied, and the results showed that the ultimate flexural strength was increased with minimal increase in beam thickness. Accordingly, the stiffness of the members was increased as one of the most important parameters. The failure modes of the timber beams strengthened with FRP presented debonding and fracture of FRP sheets, which also were the main phenomena investigated in the experiment. Plevris and Triantafillou [10] presented an experimental study on timber beams bonded by thin FRP sheets applying epoxy resins. The results proved that the retrofitted beams wrapped on the tension surface were an effective way to improve bearing capacity, stiffness, and ductility of elements. Moreover, old beams strengthened by three-layer CFRP sheets were investigated; their flexural capacity was increased up to $60.3 \%$ compared with the control beam, but less than $30 \%$ stiffness in the maximum increase was observed [11]. Yang et al. [12] conducted experimental research on the flexural behavior of timber beams retrofitted with CFRP and GFRP sheets; the results showed that the ultimate flexural strength of strengthened members improved by 17.7-77.3\% compared with the control ones. The flexural behavior of CFRP-strengthened timber beams was studied, and flexural capacity increased from 39 to $61 \%$ compared with unstrengthened beams [13, 14]. Chun and Pan [15] introduced an experimental study on rectangular pine timber beams reinforced with CFRP-AFRP hybrid FRP sheets, which enhanced flexural bearing strength and stiffness, indicating increases of $18.1-62.0 \%$ and $13-21 \%$, respectively.

Furthermore, the promising nature, low cost, and effective characteristics of basalt fiber-reinforced polymer (BFRP) make it a reasonable candidate for strengthening structures. BFRP is an inorganic fiber with excellent modulus, high strength, improved strain to failure, hightemperature and good chemical resistance, prominent stability, ability to form easily, and low cost [16]. Owing to the extraordinary properties of BFRP composites, structural strengthening applications in civil engineering that use this material have been considered [17]. Limited published literature study the improvement of the flexural capacity of timber structures bonded by BFRP composites. From the extant literature, only Pilar et al. $[8,18]$ presented an experimental and numerical study on the flexural behavior of timber beams strengthened by CFRP and BFRP. Beams retrofitted with CFRP or BFRP have higher stiffness than beams without strengthening, implying that such reinforcement enhances flexural behavior in limited state service.

In this study, poplar, as an abundant wood in Xinjiang, China, was used for building structures, although poplar has poor durability and particular mechanical properties. BFRP composites have been used to strengthen wood structures to improve ultimate bearing capacity effectively. Therefore, experimental and analytical studies were conducted and investigated to understand the flexural behavior of BFRP-strengthened timber beams from poplar. The objectives were to observe the failure modes of the control and strengthened specimens, flexural behavior, ultimate strength, and the relationship of load and deflection. In addition, a comparison was conducted between experimental and analytical results to verify whether the model can be used to analyze timber flexural behavior. The findings will be fundamental reference and theoretical basis for research on poplar beams strengthened by BFRP subjected to bending moment.

\section{Experimental program}

\section{Mechanical characteristics of materials}

To test the main mechanical characteristics of poplar timber, 60 samples cut from the same batch of poplar timber were equally separated into two groups to determine the tensile and compressive strength of the timber. Furthermore, another 30 samples revealed the proportional limit stress of the timber in compression perpendicular to the grain. The tensile strength of the timber with rift grain was tested on the samples with dimensions shown in Fig. 1, following the Chinese code GB/T 1938-2009 [19]. Compressive strength was obtained from testing the specimens with dimensions $60 \mathrm{~mm} \times 20 \mathrm{~mm} \times 20 \mathrm{~mm}$ [20]. The failure loads were achieved within 1.5-2.0 min, which was based on the samples' failure. Accordingly,

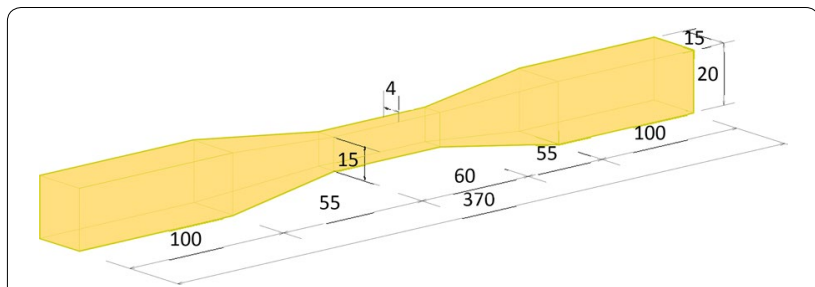

Fig. 1 Tensile testing sample with rift grain (unit: $\mathrm{mm}$ ) 
the tensile and compression strength parallel to the grain were calculated as the failure load divided by the cross-sectional area of the samples. To test the proportional limit stress in local compression perpendicular to the grain, the samples were tested under constant loading speed until failure within 1.5-2.0 min following the code of GB/T 1939-2009 [21]. The values of load and displacement were collected during testing. When the curve of load and displacement indicated a sharp turndown, the testing stopped and the values shown on the equipment were recorded. Proportional limit stress in local compression was determined as the proportional limit load divided by the cross-sectional area of the samples. Table 1 lists the three main properties of the poplar with two different moisture contents from testing.

The BFRP with unidirectional fiber illustrated in Fig. 2 was provided by Zhejiang GBF Basalt Fiber Co., Ltd., China. The BFRP composite material had a high tensile strength of $2303 \mathrm{MPa}$, an elastic modulus of $105 \mathrm{GPa}$, an elongation of $2.18 \%$, and a thickness of $0.121 \mathrm{~mm}$. The properties of the BFRP were tested and authorized by the Chinese Chemical Building Materials Testing Center. The primer (YZJ-CD) and saturant (YZJ-CQ) produced by Wuhan Changjiang Reinforcing Techniques Co., Ltd. were 2-mm-thick all-purpose epoxy resins used to bond BFRP sheets to the surface of the wooden beams. The primer penetrates the beams of cementitious substrates

Table 1 Main mechanical characteristics of poplar

\begin{tabular}{lllll}
\hline Item & $\begin{array}{l}\text { Moisture } \\
\text { content } \\
(\%)\end{array}$ & $\begin{array}{l}\text { Tensile } \\
\text { strength } \\
(\mathbf{M P a})\end{array}$ & $\begin{array}{l}\text { Compressive } \\
\text { strength }(\mathrm{MPa})\end{array}$ & $\begin{array}{l}\text { Proportionality } \\
\text { limit stress } \\
(\mathrm{MPa})\end{array}$ \\
\hline$\sigma_{9.1}$ & 9.1 & 115.6 & 36.9 & 6.1 \\
$\sigma_{12.0}$ & 12.0 & 110.6 & 31.6 & 5.3 \\
\hline
\end{tabular}

and provides a high-bond base coat for BFRP laminates, whereas the saturant encapsulates BFRP sheets and provides high performance for laminates.

\section{Details of specimens and experimental setup}

To achieve the objectives of the study, 10 timber beams with rectangular cross-sections, four for control beams, and six beams strengthened by BFRP, were cut by plain sawing from poplar. Based on the code of standard for test methods of timber structures [22], the timber beams were $50 \mathrm{~mm}$ wide by $100 \mathrm{~mm}$ high by $1900 \mathrm{~mm}$ long. The longitudinal direction of the beams was parallel to the grain direction. Six timber beams strengthened by BFRP were separated into three batches on the basis of three different strengthening configurations, which were BFRP strips that were $50 \mathrm{~mm}$ wide and 1100,1700 , and $1900 \mathrm{~mm}$ long. The direction of fiber was parallel to the beam length. Each batch had two specimens with identical bonding patterns. Based on research of the flexural behavior of timber beams, all the strengthened specimens were bonded with BFRP sheets at the bottom. For the comparison of strengthening effectiveness, three different strengthening configurations were used in the BFRP sheets for four control beams; the labels were from DBL-01 to DBL-04. Furthermore, three groups of strengthened beams were labeled JGL(B50-1100)-01\&02, JGL(B50-1700)-01\&02, and JGL(B50-1900)-01\&02. The details of strengthening configurations are shown in Fig. 3.

To bond BFRP to the bottom surface of the timber specimens, four main procedures were conducted. First, a machine was used to smoothen the beam surface and remove sawdust settled on the beam. Second, the bottom surface was coated with mixed premier epoxy using a roller. The primer was hardened for

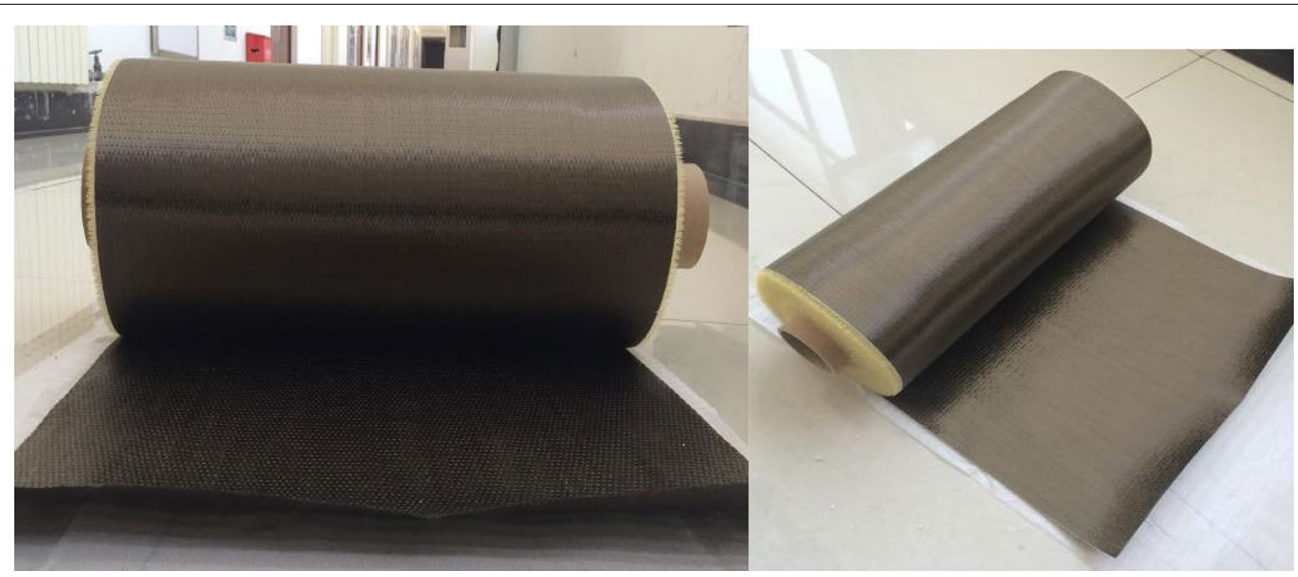

Fig. 2 Illustration of BFRP 

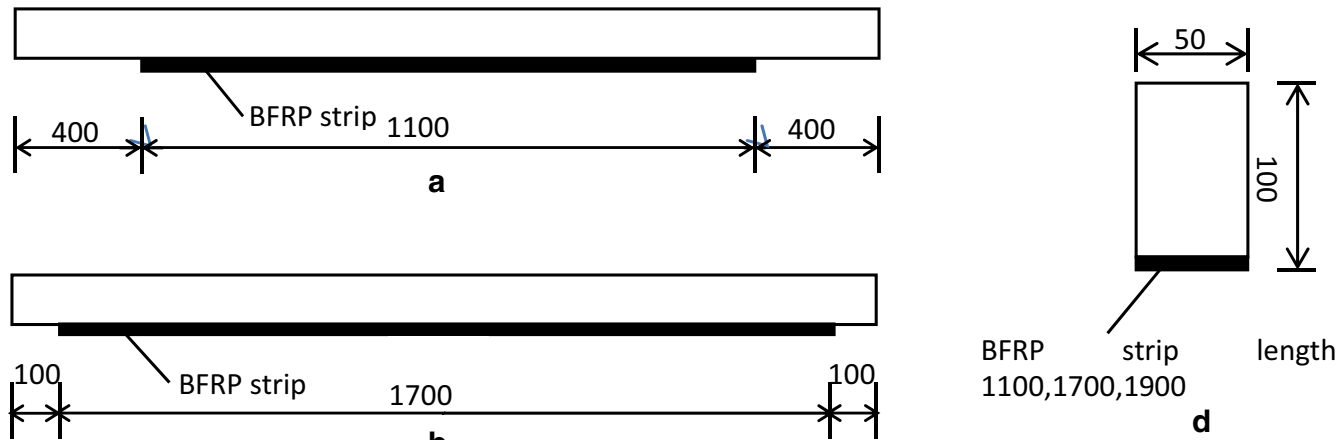

b
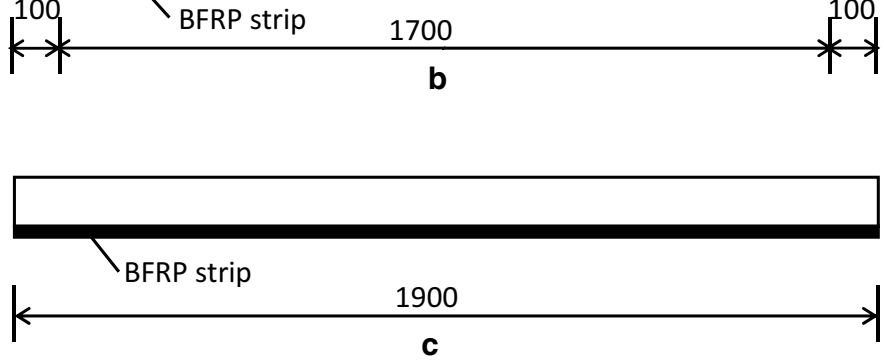

Fig. 3 Details of strengthening configurations: a JGL(B50-1100)-01\&02; b JGL(B50-1700)-01\&02; c JGL(B50-1900)-01\&02; d cross-sections of all strengthened beam (unit: $\mathrm{mm}$ )

$30 \mathrm{~min}$, after which a layer of saturant was used to cover the primer. Third, the saturated bottom surface of the specimens was covered with BFRP sheets. The epoxy resins were squeezed to wet the fiber, and the entrapped air was removed using a rigid plastic roller. Finally, the fiber was coated with a thin layer of saturant. Figure 4 illustrates the main strengthening procedure.

The timber specimens were loaded using a hydraulic servo system of $200 \mathrm{kN}$ capacity with four-point bending in the structural laboratory of Xinjiang University, China. The loading on the spreader beam was divided into two equal loads on the timber beams. The load values were loaded automatically by hydraulic actuator with $2.1-\mathrm{kN}$ intervals, which were increased continuously and evenly until the beams failed.

To measure the strain distribution and the height of the beam, for all the tested beams, 10 were arranged to be bonded on the two sides of the surface of mid-span. Using three linear variable differential transducers (LVDTs), the vertical displacements of the specimens were measured at mid-span and at two points, which were $300 \mathrm{~mm}$ from the mid-span. Two dial indicators with $0.01 \mathrm{~mm}$ accuracy were located on top of both ends of the beams. Load, deflection, and strain gauge data were automatically collected and recorded using a static data measurement system (DH3816). Figure 5 illustrates in detail the testing setup and instrumentation.

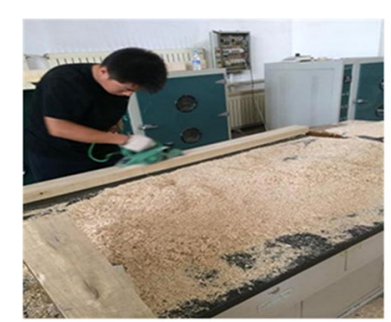

a

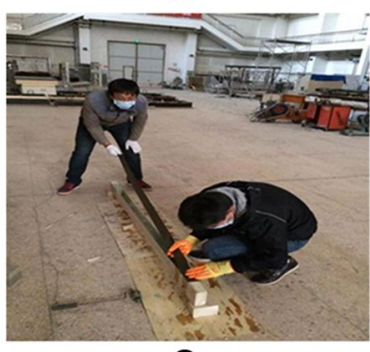

C

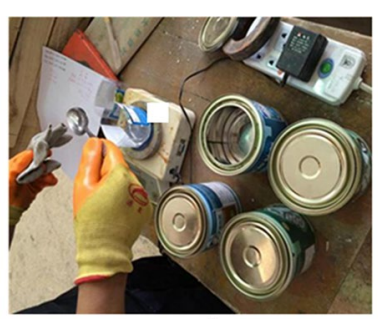

b

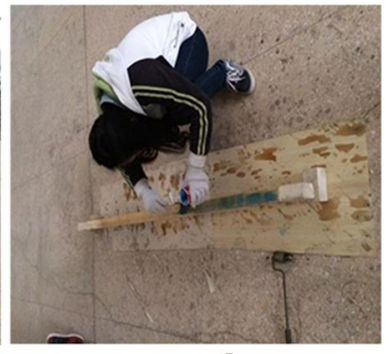

d

Fig. 4 Main procedure of strengthening BFRP: a smoothed surface of beam, $\mathbf{b}$ primer mixed, $\mathbf{c}$ primer and saturant painted, and $\mathbf{d}$ FRP bonded

\section{Testing results}

\section{Summary of testing results}

Table 2 presents the ultimate load and mid-span deflection of control beams and BFRP-strengthened beams. The increment of the ultimate load and deflection of reinforced specimens compared with the control beams were also included. Table 2 indicates that although the four control beams had the same dimension and were 


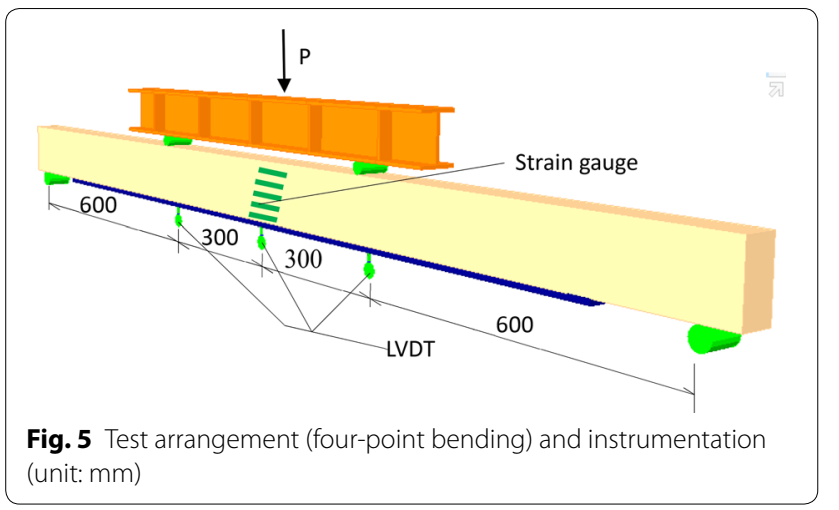

subjected to bending at the same loading procedure, the ultimate load and the maximum deflections were noticeably varied because of the natural characteristics of the timber. The ultimate load of DBL-01 and DBL-02 was $10.5 \mathrm{kN}$, but that of DBL-03 and DBL-04 was $8.4 \mathrm{kN}$. Furthermore, the highest deflection of $32.4 \mathrm{~mm}$ was in the beam of DBL-04. However, for easy comparison with BFRP-strengthened beams, the average ultimate load and maximum deflection of the four control beams used the values of $9.45 \mathrm{kN}$ and $29.72 \mathrm{~mm}$, respectively.

For BFRP-strengthened timber beams, the ultimate load and deflection had increased under different strengthening configurations. However, the bending resistance of the timber beams externally bonded by BFRP was increased, resulting in the enhancement of the maximum deflection at the ultimate state. Table 2 clearly shows that the deflection of JGL(B50-1900)-01\&02 presented the highest ultimate load of $16.8 \mathrm{kN}$ and a deflection of $62.44 \mathrm{~mm}$. This result indicates a $77.8 \%$ and $110.1 \%$ increase in ultimate load and deflection, respectively, compared with the control ones. The strengthened beams of JGL(B50-1100)-01\&02 showed the weakest strengthening effectiveness, in which the increases were $33.3 \%$ and $20.6 \%$ in ultimate load and deflection, respectively. As expected, JGL(B50-1700)-01\&02 showed intermediate improvement. Therefore, the full-length BFRP sheets bonded on the bottom surface of the beams JGL(B50-1900)-01\&02 contributed the highest improvement and provided the best strengthening effectiveness. This finding explains that BFRP bonding length is a crucial parameter that affects the strengthening contribution to retrofitted specimens. Furthermore, bonding BFRP on the bottom of the beams decreased the effect of the deficiencies of the beams to improve the capacity of the timber beams.

\section{Failure modes of tested specimens}

During the testing procedures, four control timber beams were not investigated for cracking, and the sound of failure "cracking" was heard at the $0-4.2 \mathrm{kN}$ loads. Here, the beams were in an elastic state in the load-deflection relationship. At the loads of $4.2-8.4 \mathrm{kN}$, the deflection of the tested specimens were increasing, accompanied by a splitting sound inside the timber fiber; here, the beams were in the plastic stage. At the ultimate stage, four control timber beams exhibited different ultimate loads, which were $8.4 \mathrm{kN}$ for DBL-03 and DBL-04 and $10.5 \mathrm{kN}$ for DBL-01 and DBL-02. From the experimental investigation, the cracking occurred immediately after loud "cracking" sounds were heard from the failure part of the beams. Here, we emphasize that the flexural capacity of the timber was lower than that expected because of the knots and tiny cracks in timber. Therefore, failure investigated from the tested beams almost appeared at the knots located in the mid-span. Interestingly, britthe failure occurred from the bottom and immediately expanded into the whole cross-section. However, considering the structural mechanic principle and that

Table 2 Summary of ultimate load and deflection of tested specimens

\begin{tabular}{|c|c|c|c|c|c|c|}
\hline Beam & $\begin{array}{l}\text { Ultimate load } \\
\text { (kN) }\end{array}$ & $\begin{array}{l}\text { Average of ultimate } \\
\text { load }(k N)\end{array}$ & $\begin{array}{l}\text { Increase in ultimate } \\
\text { load (\%) }\end{array}$ & Deflection (mm) & $\begin{array}{l}\text { Average } \\
\text { of deflection }(\mathrm{mm})\end{array}$ & $\begin{array}{l}\text { Increase } \\
\text { in deflection } \\
(\%)\end{array}$ \\
\hline DBL-01 & 10.5 & 9.45 & - & 25.45 & 29.72 & - \\
\hline DBL-02 & 10.5 & & & 28.77 & & \\
\hline DBL-03 & 8.4 & & & 32.29 & & \\
\hline DBL-04 & 8.4 & & & 32.4 & & \\
\hline JGL(B50-1100)-01 & 12.8 & 12.6 & 33.3 & 36.55 & 35.85 & 20.6 \\
\hline JGL(B50-1100)-02 & 12.4 & & & 35.15 & & \\
\hline JGL(B50-1700)-01 & 14.3 & 14.7 & 55.6 & 39.46 & 39.78 & 33.8 \\
\hline JGL(B50-1700)-02 & 15.1 & & & 40.1 & & \\
\hline JGL(B50-1900)-01 & 16.3 & 16.8 & 77.8 & 60.65 & 62.44 & 110.1 \\
\hline JGL(B50-1900)-02 & 17.3 & & & 64.23 & & \\
\hline
\end{tabular}


compressive strength is lower than tensile strength, the top of the beams should be crushed instead of the tension fracture. This phenomenon can be explained by the timber fiber of the bottom reaching the ultimate strain or deficiencies (knots and cracks) in the lower section of the beam, resulting in stress concentration and the failure that occurred at the bottom of the control specimens. Figure 6 illustrates the typical failure of the control timber beams.

In the timber specimens retrofitted with BFRP, two beams in each batch exhibited a slight difference in flexural behavior during the test and at the ultimate state. Therefore, the following descriptions of the behavior of the specimens will be summarized by the overall condition of two beams in each batch. For JGL(B50-1100)01\&02, no distinct changes occurred when the load ranged from 0 to $10.5 \mathrm{kN}$, but the deflection was found to increase and the sound of timber fiber breaking was heard when the load reached $10.5 \mathrm{kN}$. At ultimate loading, the specimens underwent brittle rupture with a loud sound of a broken beam. Here, the two beams almost had the same failure modes, in which the timber beam was broken from the timber knots with the BFRP strip still bonded to the bottom of the beams, which is shown in Fig. $7 \mathrm{a}$ and b. The "cracking" sound of JGL(B50-1700)$01 \& 02$ was heard when the loading reached 10.5 to $12.6 \mathrm{kN}$, and deflection was increasing obviously. Furthermore, the beam JGL(B50-1700)-01 was broken at the ultimate loading of $14.3 \mathrm{kN}$ which resulted in the fracture of BFRP sheet fracture. However, JGL(B50-1700)-02 was loaded to become two parts, with BFRP debonding from the bottom surface of the beam at a load of $15.1 \mathrm{kN}$. These two beams exhibited two different failure conditions, which are shown in Fig. $7 \mathrm{c}$ and d. However, both specimens of JGL(B50-1900)-01\&02 indicated the same flexural behavior during testing and at the ultimate state. The deflection of the beams was growing as the loading was increased, and it was accompanied by the "cracking" sound of the timber fiber. When the load reached 16.8 $\mathrm{kN}$, the specimen was broken and the BFRP sheet ruptured and debonded from the beam, as shown in Fig. 7e. From the testing of the strengthened timber beams, although BFRP sheets helped improve the strength of the timber beams, these specimens were broken because of the deficiencies (such as knots) and mechanical characteristics of poplar timber.

\section{Flexural performance of tested specimens}

1. Verification of the "plane section remains plane" assumption

As previously mentioned, 10 strain gauges were bonded to two sides of the tested beams along with the cross-section height. The collected results implied that the strain was basically linear over the height of the cross-section for the control and BFRP-strengthened beams. During the loading, the neutral axis of the control shifted to the upper half of the cross-section shown in Fig. 8a, but the neutral axis of the strengthened beams shifted to the lower half of the cross-section shown in Fig. 8b. A comparison of the strain distribution of the control beam and JGL(B50-1900) at ultimate stage revealed that the tensile and compressive strain of JGL(B50-1900) was greater that of the control beam. However, at the same loading level such as $10 \mathrm{kN}$, the strain $3800 \mu \varepsilon$ of JGL(B50-1900) was lower than $4600 \mu \varepsilon$ of the control beam. This result verified that BFRP strengthened the timber beam to improve the capacity of the strengthened specimen.

In addition, the strengthened beams in the other two batches exhibited the same phenomenon of the neutral axis, and the relationship of the strain and the cross-section height with the beam of JGL(B50-1900), illustrated in Fig. 8b.

Therefore, testing verification indicates that the assumption "plane section remains plane" satisfied
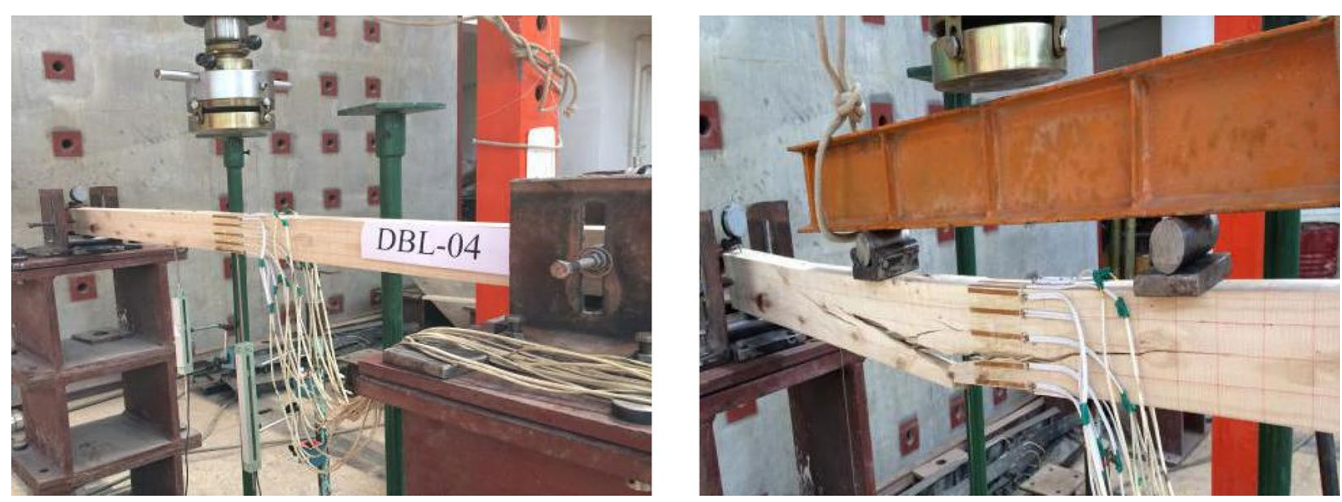

Fig. 6 Typical failure of the control timber beams 


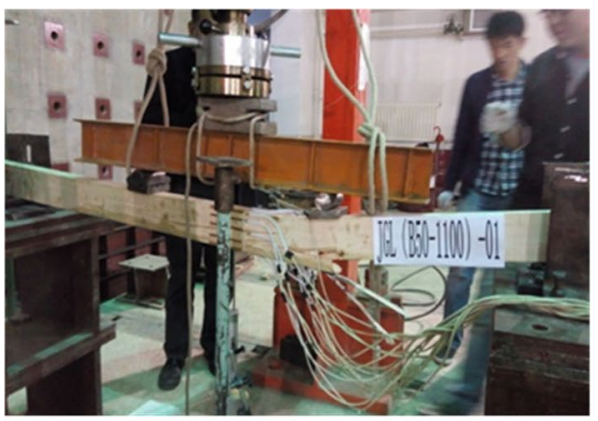

a

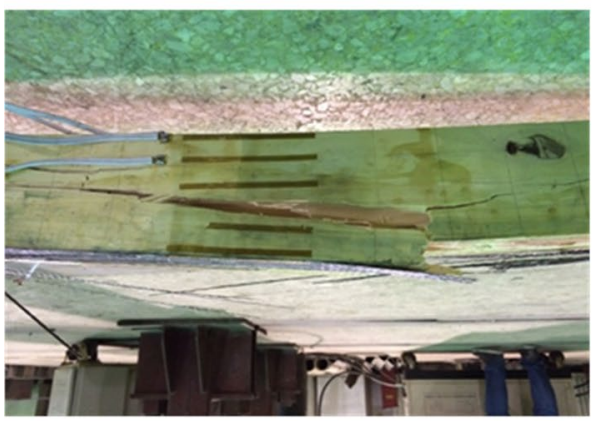

C

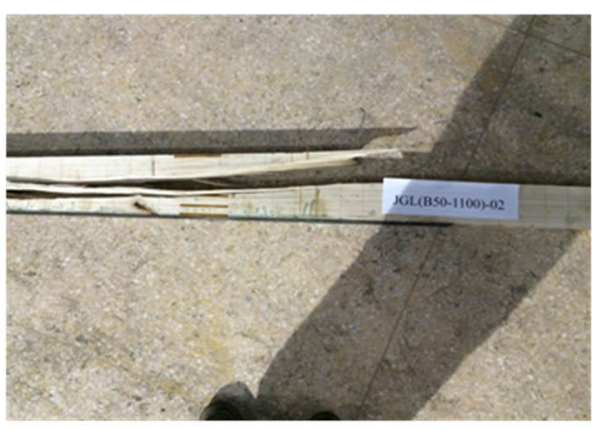

b

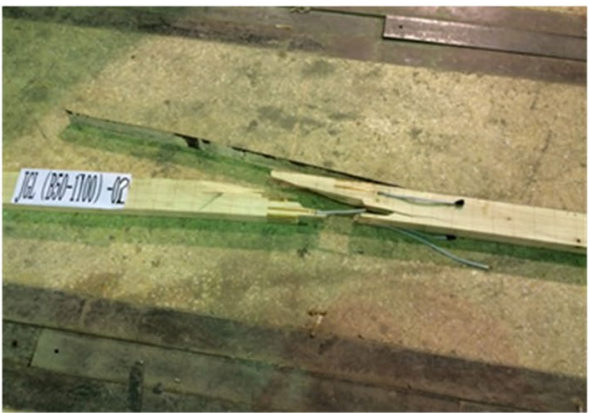

d

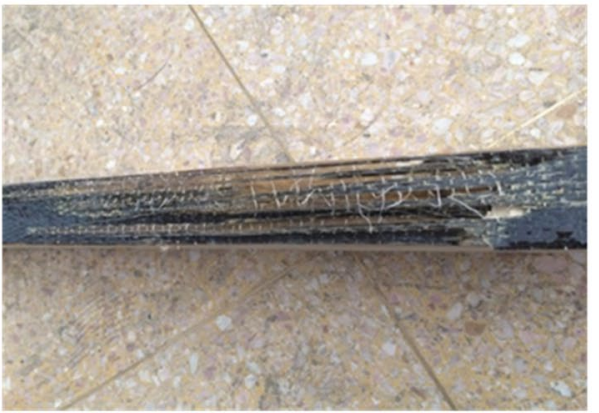

e

Fig. 7 Typical failure of tested beams: a for JGL(B50-1100)-01; b for JGL(B50-1100)-02; c for JGL(B50-1700)-01; d for JGL(B50-1700)-02; e for JGL(B50-1900)-01\&02

bending theory of the normal section. Each test showed that the strain across the cross-section of the control and strengthened timber beams was linear in the entire loading process.

\section{Load-deflection response}

Figure 9 reveals the load-deflection relationship at the mid-span of the control and strengthened timber beams. Table 2 shows the load-bearing capacity and corresponding deflection of all timber specimens. From the overall trend of load-deflection curves, in the same loading value, such as $6.3 \mathrm{kN}$, the deflections of the control beam, JGL(B50-1100), JGL(B50$1700)$, and JGL(B50-1900) were 18.9, 13.55, 13.75, and
$13.2 \mathrm{~mm}$, respectively. Therefore, compared with the control beams, the stiffness of strengthened beams was relatively improved, and the deflection was decreased accordingly at the same loading state. Besides, the strengthened beams almost had the same load-deflection trend when the loading ranged from $0-8.4 \mathrm{kN}$. After this stage, the beams with different BFRP bonding lengths began to express different conditions. JGL(B501900), with the longest bonding length, presented the highest deflection with the value of $62.44 \mathrm{~mm}$ and the greatest bearing capacity at the ultimate state. Furthermore, the loading and deflection of JGL(B50-1700) were higher than those of JGL(B50-1100). Based on 

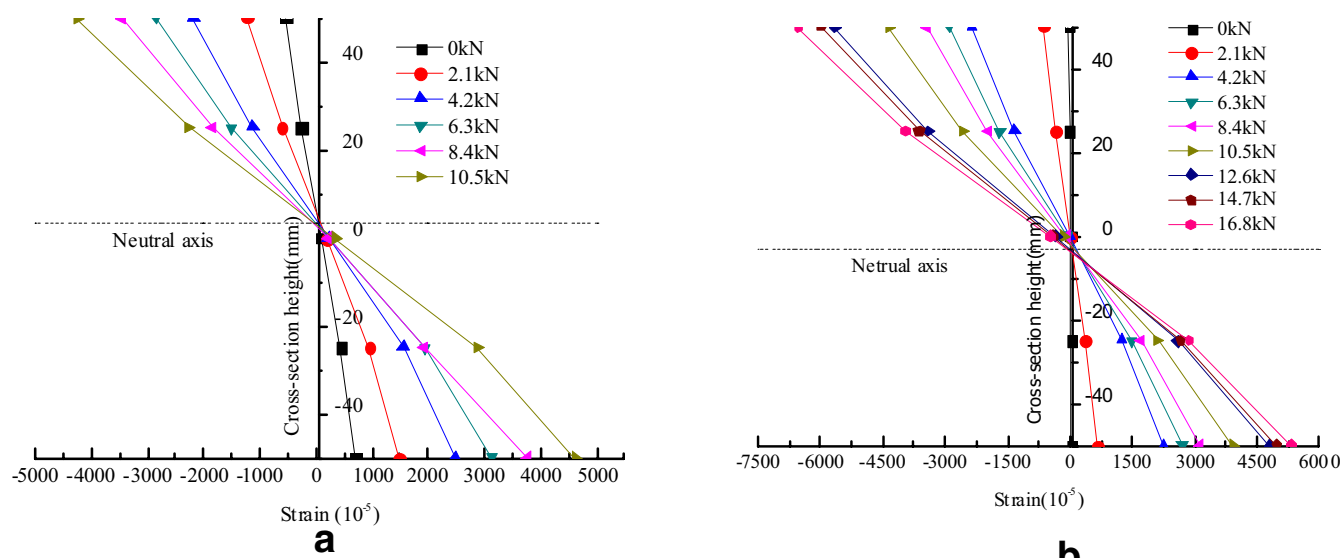

b

Fig. 8 Curves of strain versus cross-section height a for control beam; b for JGL(B50-1900)

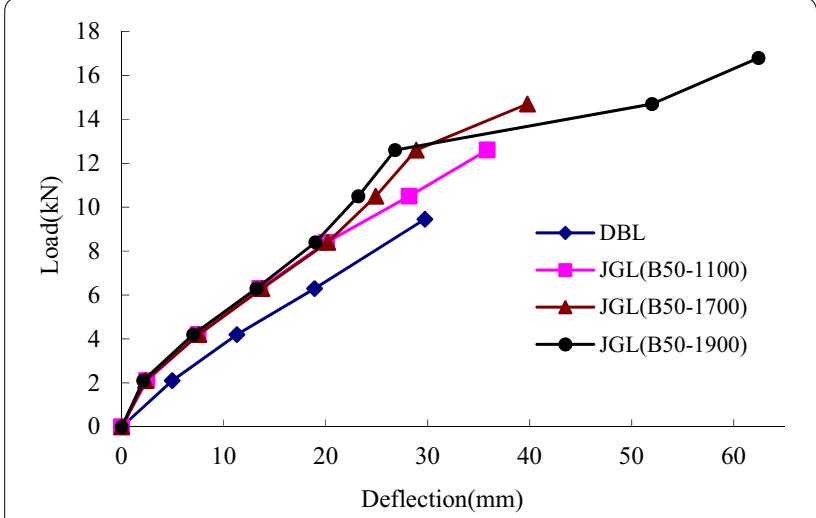

Fig. 9 Curves of load-deflection of all beams

the different strengthening configurations, bonding length was a crucial parameter that affected flexural strengthening effectiveness. At the initial stage of loading, BFRP provided stiffness to the timber beams, and no debonding occurred. As the loading increased until the ultimate load, BFRP strips of JGL(B50-1100)-01\&02 showed two different failure patterns of debonding and rupture, but the beams failed at the ultimate state. Therefore, although BFRP strips can improve a certain capacity of timber beam, they cannot prevent the beams from rupturing. The same phenomenon occurred in JGL(B50-1700)-01\&02. However, at the ultimate state, the load and deflection increased with the increase in BFRP strip length. The beam of JGL(B50-1900) had a different failure mode, in which the BFRP strip was fractured without any debonding at the ultimate state. This result may be attributed to the beam support that suppressed the BFRP strip to avoid debonding. Therefore, the beam had the highest bearing capacity and deflection at the mid-span, because the BFRP strip gave full play to the strain applied in improving the stiffness of the strengthened beam.

\section{Finite element analysis (FEA) simulation}

To understand further the behavior of timber beams bonded with BFRP composite, the general FEA software ANSYS was used to build a 3D analytical model to predict the bending behavior of timber beams retrofitted with BFRP sheets, in contrast with the experimental results. The following section summarizes the detailed modeling approaches.

Owing to the orthotropic characteristics of timber [23], 3D solid elements (SOLID45) shown in Fig. 10a were used to represent timber. SOLID45 is an eight-node element with three translational degrees in the nodal $x, y$, and $z$ directions of freedom per node to match the orthotropic properties of timber specimens, which are large deflection, plasticity, swelling, creep, stress stiffening, and large strain capabilities [24]. Furthermore, the unidirectional BFRP sheets were modeled with 3D membrane elements (SHELL41) in Fig. 10b. The element consists of three degrees of freedom at each node: translations in the nodal $x, y$, and $z$ directions. Thus, no constraint equations were applied to couple the timber elements and BFRP elements, meaning that the two elements had compatibility of in degrees of freedom. Other testing programs also recorded "perfect bond" properties in FRP-strengthened timber beams $[6,23]$. SOLID45 also was used for the steel plates as support and at the points of loading application in the timber beam modes.

For the timber material, the surfaces are categorized into three geometric planes of reference that indicate the type of surface uncovered after a cut has been made. The three reference axes are longitudinal, radial, and tangential, as shown in Fig. 11a. For simplified modeling of 
wood, three orthogonal axes of $\mathrm{L}\left(\mathrm{X}_{1}\right), \mathrm{T}\left(\mathrm{X}_{2}\right)$, and $\mathrm{R}\left(\mathrm{X}_{3}\right)$ were adopted to present $3 \mathrm{D}$ fiber of the timber, as shown in Fig. 11b. Therefore, as an orthotropic material, three directions of the timber have different elastic characteristics, which are represented by the elastic modulus $E_{\mathrm{i}}$, Poisson's ratio $\mu_{\mathrm{ij}}$, and shear modulus $G_{\mathrm{ij}}$, as shown in Table 3. An elastic orthotropic constitutive relationship for timber elements may be developed [23] as

$$
\begin{aligned}
& \{\sigma\}=[D]\{\varepsilon\}, \\
& \{\varepsilon\}=\left\{\varepsilon_{\mathrm{R}} \varepsilon_{\mathrm{L}} \varepsilon_{\mathrm{T}} \gamma_{\mathrm{LR}} \gamma_{\mathrm{LT}} \gamma_{\mathrm{RT}}\right\}^{\mathrm{T}},
\end{aligned}
$$

where the stress vector $\{\sigma\}=$ in which $\sigma_{i}$ is the normal stress in the radial (R), longitudinal (L), and tangential
(T) directions, and $\tau_{i j}$ is the shear stress in the $i j$ direction; $[D]$ is the orthotropic stiffness matrix $[D]=\left[C_{i j}\right]$ in which $C$ is the elastic constant and $\{\varepsilon\}$ is the corresponding strain vector. To ensure the positive definition of the stiffness matrix, the determinant of all submatrices of the orthotropic stiffness matrix must be positive [24]. The orthotropic stiffness matrix was built with the mechanical properties of the timber members shown in Table 3. The BFRP composite was assumed to exhibit a linear elastic response. The tensile modulus of the BFRP studied at present is $105 \mathrm{GPa}$. A Poisson's ratio of 0.3 was applied for the BFRP composites. Apart from the above parameters considered for non-linear elastic-plastic analysis, an appropriate multilinear kinematic of yield criterion
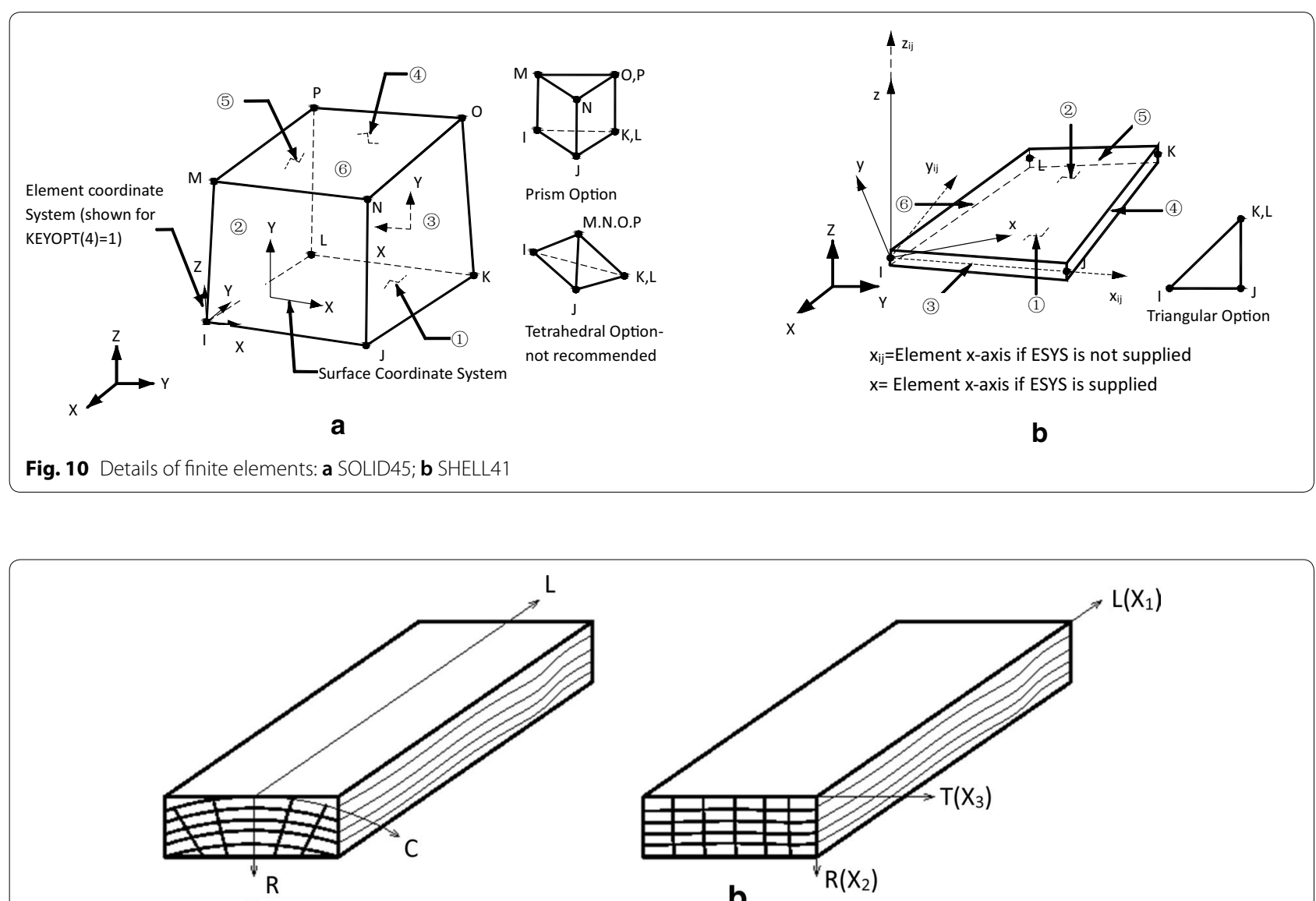

a

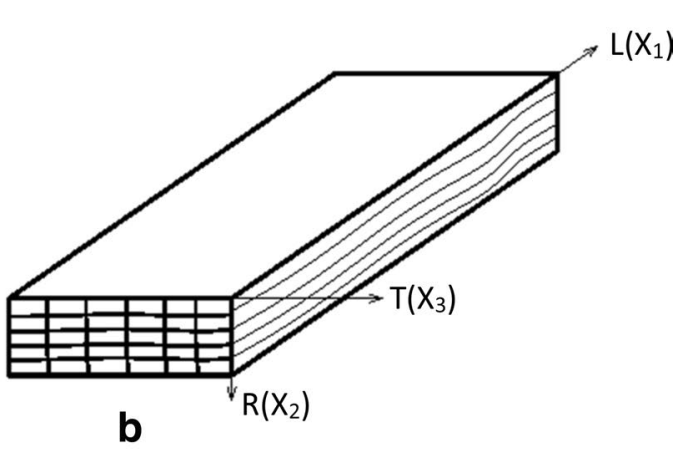

Fig. 11 Orthotropic property of wood [27]: a three-dimensional orientation of wood; $\mathbf{b}$ orthogonal Cartesian axes

Table 3 Elastic constant parameters of poplar [28]

\begin{tabular}{lllllllll}
\hline $\begin{array}{l}E_{\mathrm{R}} \\
\mathrm{MPa}\end{array}$ & $\begin{array}{l}E_{\mathrm{L}} \\
\mathrm{MPa}\end{array}$ & $\begin{array}{l}E_{\mathrm{T}} \\
\mathrm{MPa}\end{array}$ & $\mu_{\mathrm{RL}}$ & $\mu_{\mathrm{LT}}$ & $\mu_{\mathrm{RT}}$ & $G_{\mathrm{RL}}$ & $\begin{array}{l}G_{\mathrm{LT}} \\
\mathrm{MPa}\end{array}$ \\
\hline 800 & 9431 & 610 & 0.2 & 0.47 & 0.43 & 915 & $\begin{array}{l}G_{\mathrm{RT}} \\
\mathrm{MPa}\end{array}$ \\
\hline
\end{tabular}


had to be used with the plastic strain developing instantaneously. Flow rule can be ascertained with the multilinear kinematic selected. In particular, the generalized Hill yield criterion [25] is an anisotropic model that accounts for different yield strength in 3D coordinates of the materials, as well as the different yield strengths in tensile and compressive states [26], which is close to the timber properties. Therefore, the generalized Hill yield criterion was adopted in the present modeling. Furthermore, two essential assumptions have been considered in FEA for simulating timber beam accurately. Timber is homogeneous and continuous without knots, cross-grain, and tiny cracks. Furthermore, environmental factors, such as temperature and moisture that affect the behavior of timber specimens were not considered.

Figure 12 gives the FEA model of the timber beam. The solid elements of SOLID45 were used to represent the wood specimens. The 3D elements with eight nodes include a translational degree of freedom (DOF), which was suitable for the orthotropic properties of the timber members that were described in the previous part. Furthermore, the SHELL41 of the 3D membrane elements was preferred to simulate BFRP sheets with unidirectional characteristics. Boundary and loading conditions were applied to the tested timber beam model by restraining the essential nodes to stand for the simply supported situation. No slippage and interface elements were considered between timber beam and BFRP and between timber and supporting plates. Other experimental programs also reported "perfect bond" characteristics in FRP-strengthened timber beams [6, 29]. In addition, the nodes' deformation compatibility of DOF occurred between the elements. Mesh density normally affects the convergence and accuracy of the modeling results; thus, the optimum mesh size of $25 \mathrm{~mm}$ of hexahedral mapping was verified [30] and adopted for the numerical study of the specimens.

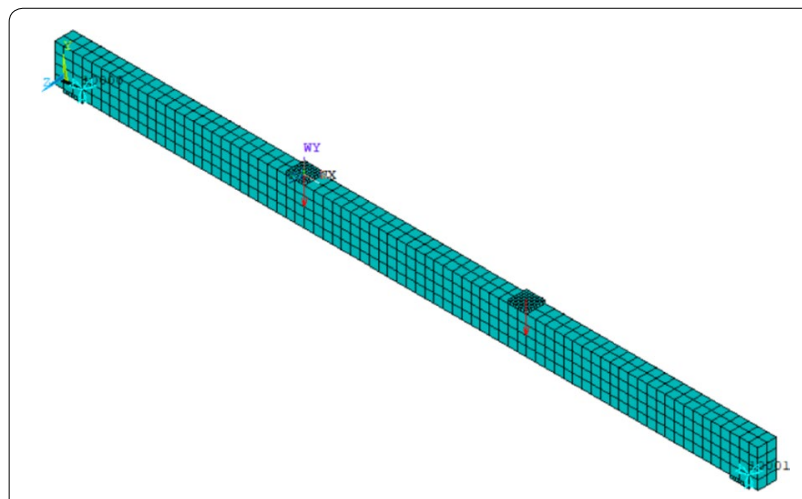

Fig. 12 FEA model of timber beam

\section{Results of FEA simulation}

\section{Load-deflection response}

Figure 13a describes the relationship of load displacement of unstrengthened beam DBL at the mid-span. From the curves of experimental and analytical values, the results of the predicted load-deflection of the specimen are in considerable agreement with experimental ones. In the load range of $0-4.2 \mathrm{kN}$, the curves illustrate identical trends with the same slope of the straight lines, which can be considered the stage of elasticity. After this state, the timber beam shifts to the plastic deformation stage within the load of $4.2-7.5 \mathrm{kN}$, in which the predicted displacement is slightly lower than that of the testing results at the same load condition. In the last stage close to failure, the maximum displacement of the numerical value is $38.07 \mathrm{~mm}$, which is slightly higher than the experimental value of $36.8 \mathrm{~mm}$. From the comparison of the entire procedure of analysis and experiment, the load-displacement trends are highly identical, demonstrating that the

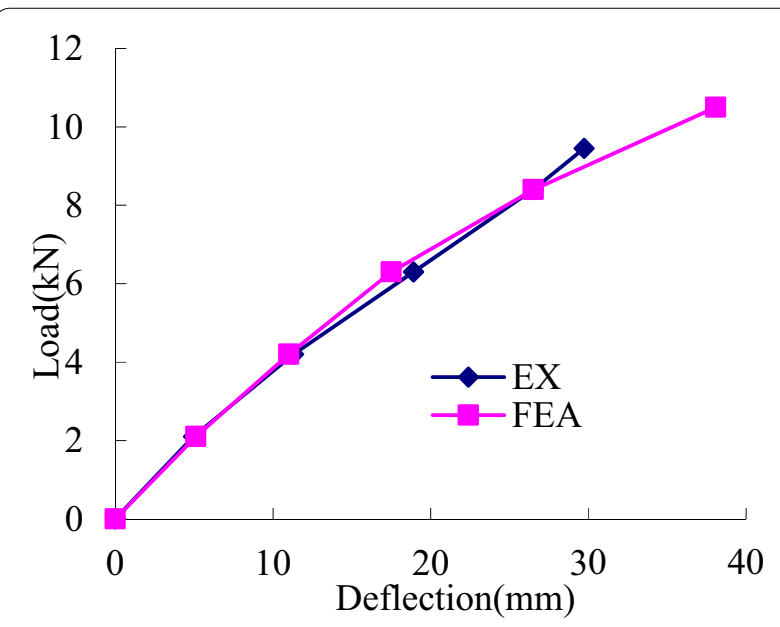

a

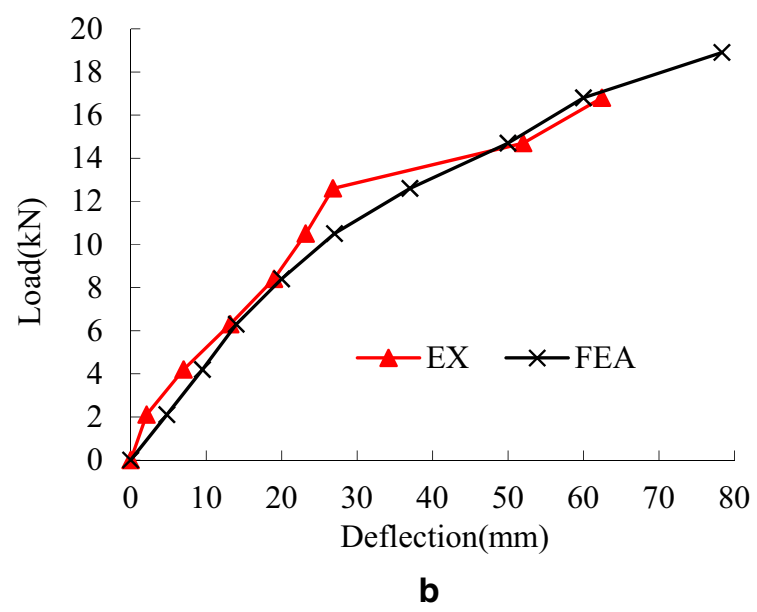

Fig. 13 Curves of load-deflection of: a DBL; b JGL(B50-1900) 
FEA model can closely simulate the flexural behavior of timber beams. Moreover, the parameters adopted in the modeling are suitable to predict the overall testing process. In addition to investigating the control specimen, Fig. 13b presents the curves of load-displacement of JGL(B50-1900) strengthened by BFRP strips that measure $50 \mathrm{~mm}$ wide and $1900 \mathrm{~mm}$ long. The predicted ultimate load is $18.9 \mathrm{kN}$, which is higher than the experimental value of $16.8 \mathrm{kN}$. Accordingly, the displacement is also greater than the results of testing. Under the same condition of the control beam, the trend of tested load-displacement of the strengthened beam indicates close agreement with analytical results at the load range of $0-8.4 \mathrm{kN}$ in the elastic stage. In the loading from 8.4 to $13.5 \mathrm{kN}$, plasticity appears in the specimen, and the predicted displacement is slightly higher than that of the experimental results. However, the numerical trend of displacement changes from higher to lower than that of the testing results after the loading of $13.5 \mathrm{kN}$. In the ultimate stage, the highest displacements of predicted and testing values are 78.34 and $68.68 \mathrm{~mm}$, respectively. Although a nonsignificant deviation of deflection occurs between analytical and experimental results, the transformation law of curves has a relatively similar trend in the overall procedure.

\section{Ultimate load and deflection of mid-span}

On the basis of extracting the numerical simulation data from modeling, the ultimate load, the deflection of mid-span, and the increment of bearing capacity of each group were achieved and shown in Table 4.

Table 4 Ultimate bearing capacity and deflection of midspan by FEA

\begin{tabular}{lllll}
\hline Beam & $\boldsymbol{P}_{\mathbf{u}}$-FEA $(\mathrm{kN})$ & $\boldsymbol{F}_{\mathbf{u}}-\mathrm{FEA}(\mathbf{m m})$ & $\begin{array}{l}\text { Increment } \\
\text { of } \boldsymbol{P}_{\mathbf{u}}(\%)\end{array}$ & $\begin{array}{l}\text { Increment } \\
\text { of } \boldsymbol{f}_{\mathbf{u}}(\%)\end{array}$ \\
\hline DBL-01 & 10.5 & 38.07 & $/$ & $/$ \\
JGLB50-1100 & 14.7 & 63.54 & 40 & 67 \\
JGLB50-1700 & 16.8 & 66.12 & 60 & 74 \\
JGLB50-1900 & 18.9 & 78.34 & 80 & 106 \\
\hline
\end{tabular}

$P_{\mathrm{u}}$ is ultimate load; $f_{\mathrm{u}}$ is deflection of mid-span
Beam DBL-01, which was unstrengthened, presented the lowest load and deflection. However, all the strengthened beams increased the ultimate load and deflection. Interestingly, the increments of the beam strengthened by full-length BFRP strips show the best improvement either in ultimate capacity or mid-span deflection, such that the increments are 80 and 106\% compared with the non-strengthened beam. The beams with 1100- and 1700-mm-long BFRP strips indicate increases of 40 and $60 \%$ in ultimate load and 67 and $74 \%$ in mid-span deflection, respectively. The same strengthening effectiveness and improvement tendency of capacity and deflection of the beams were investigated from experiments and analysis, indicating that the best strengthening configuration is the full-length BFRP strips.

Furthermore, Table 5 shows the comparison of the ultimate load and deflection of all the specimens between experimental and FEA results. The ratios of testing and analytical values of strengthened timber beams in ultimate load and deflection of mid-span are greater than 1 , clearly demonstrating that the results from FEA were overestimated compared with the values from the testing. The highest ratios of ultimate load and deflection from JGB50-1100 are 1.167 and 1.410, respectively, showing a certain error similar to the other two strengthened beams. The analytical values from the specimen without strengthening are in agreement with the ones from the experiment. Therefore, the properties of timber and related parameters considered in the FEA modeling were relatively reasonable and feasible. However, distinct errors appeared in all the strengthened specimens. One possible reason for this result is that the reasonable strain-stress relationship of BFRP and the slippage relationship between timber and BFRP were not involved in the modeling, thereby consolidating the bonding of the timber and BFRP fiber. Therefore, all the analytical values of ultimate load are higher than those of testing. The FEA modeling of this study can roughly simulate the flexural behavior of strengthened beams, and the results can be used as reference. However, the precise modeling needs to be modified and developed on the basis of reasonable parameters of BFRP and contact elements with the accurate relationship between BFRP and timber.

Table 5 Comparison of experimental and FEA results

\begin{tabular}{lllllll}
\hline Beam & $\boldsymbol{P}_{\mathrm{u}}$-FEA $(\mathbf{k N})$ & $\boldsymbol{P}_{\mathrm{u}}$-EX $(\mathbf{k N})$ & $\boldsymbol{f}_{\mathrm{u}}$-FEA $(\mathbf{m m})$ & $\boldsymbol{f}_{\mathrm{u}}$-EX $(\mathbf{m m})$ & $\boldsymbol{P}_{\mathrm{u}}$-FEA/ $\boldsymbol{P}_{\mathrm{u}}$-EX & $\boldsymbol{f}_{\mathrm{u}}$-FEA/f $\boldsymbol{f}_{\mathrm{u}}$-EX \\
\hline DBL-01 & 10.5 & 10.5 & 38.07 & 36.8 & 1.000 & 1.035 \\
JGLB50-1100 & 14.7 & 12.6 & 63.54 & 45.06 & 1.167 & 1.410 \\
JGLB50-1700 & 16.8 & 14.7 & 66.12 & 49.78 & 1.143 & 1.328 \\
JGLB50-1900 & 18.9 & 16.8 & 78.34 & 64.06 & 1.125 & 1.223 \\
\hline
\end{tabular}

$P_{\mathrm{u}}$ is ultimate load; $f_{\mathrm{u}}$ is deflection of mid-span 


\section{Neutral axis}

Figure 14 discloses the comparison of strain versus crosssection height for the control beam DBL under the 8.4$\mathrm{kN}$ load and for the strengthened beams with different strengthening configurations under the $10.5-\mathrm{kN}$ load. For the control beam, the neutral axis, based on the numerical analysis and testing results, all moved toward the compression zone with minimal movement. The strain was not completely distributed in a straight line along the section, which can be judged to be in the elastic-plastic stage. In the test under the $10.5 \mathrm{kN}$ load, the neutral axis of all BFRP-strengthened beams shifted downward to the tensile zone, in which the phenomenon is opposite to the control beam. Therefore, BFRP strips can improve a certain stiffness to timber beams during loading. The strain was not completely distributed in a straight line along the section, which also can be considered in the elastic-plastic stage. Under the same load, the strain value decreased with the increase of the BFRP bonding length. The numerical analysis results were close to the experimental results to a great extent, and the simulated values were slightly less than the experimental ones. The reason for these findings is that the assumptions of FEA were mostly ideal, but the entire experimental process indicates discreteness.

\section{Conclusion}

In this study, the failure characteristics, ultimate load, deflection and strain of control beams and BFRPstrengthened beams were explored and analyzed with reference to poplars. The following main conclusions were drawn.

The average failure load of the unreinforced timber beam was $9.45 \mathrm{kN}$, and the average maximum deflection of the beam at the bottom span was $29.72 \mathrm{~mm}$. The unstrengthened beams eventually underwent brittle failure at the ultimate state owing to excessive deflection.

The assumption "plane section remains plane" remains reasonable and rational to the poplar beam retrofitted with BFRP. Moreover, the neutral axis of the beam whose bottom was reinforced had a tendency to move toward the tensile zone, with minimal movement. This result
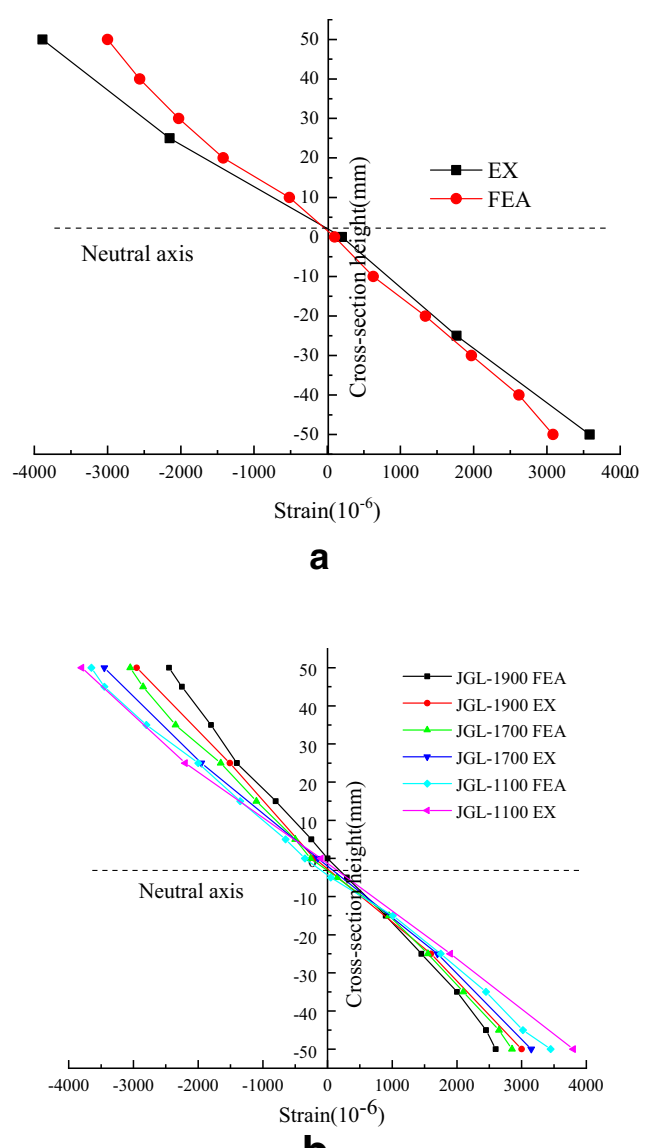

b

Fig. 14 Comparison of strain versus cross-section height: $\mathbf{a}$ for control beam; $\mathbf{b}$ for strengthened beam 
indicates that the compressive yield point of the wood was substantially lower than the tensile yield point, which was consistent with the practical situation.

In the bending test of poplar timber beams strengthened by BFRP strips of three different lengths on the bottom, the ultimate flexural capacity and deflection of the beam with 1900-mm-long and 50-mm-wide BFRP strips were higher than those of the other two strengthened beams, whose increments were 77.8 and $110.1 \%$ compared with the control beam. Therefore, among three different strengthening configurations, full-length BFRP strip bonded to timber beam can effectively and considerably improve flexural performance.

From the comparison of FEA and experimental values, the ultimate load and deflection of mid-span achieved from FEA are greater than those from testing. These findings can be used as reference for timber beams strengthened by BFRP. However, modifying and developing precise modeling with a reasonable relationship between BFRP composites and timber beams from Xinjiang poplar should be a focus of subsequent research.

\section{Abbreviations \\ BFRP: Basalt fiber-reinforced polymer; FRP: Fiber-reinforced polymer; CFRP: Carbon fiber-reinforced polymer; GFRP: Glass fiber-reinforced polymer; AFRP: Aramid fiber-reinforced polymer; FEA: Finite element analysis; LVDT: Linear variable differential transducer; DOF: Degree of freedom.}

\section{Acknowledgements}

This study was conducted with the support of Xinjiang Uygur Autonomous Region (2019D01C048) and Special Funds (CHD300102219525) for Fundamental Scientific Research Business Expenses of Central Universities.

\section{Authors' contributions}

QL planned and supervised the research. SM analyzed the experimental data and wrote the manuscript. XH drew all the figures. All authors read and approved the final manuscript.

\section{Funding}

This project was funded by Natural Science Grant of Xinjiang Uygur Autonomous Region and Fundamental Scientific Research Business Expenses of Central Universities.

\section{Availability of data and materials}

All data generated or analyzed during this study are included in this published article.

\section{Competing interests}

The authors declare that they have no competing interests.

Received: 18 January 2020 Accepted: 17 May 2020

Published online: 24 May 2020

\section{References}

1. Minjuan H (2008) Timber engineering. China Building Industry Press, Beijing (in Chinese)

2. Fang Q (1989) A study on the division of the geographical product area of populus bolleana in Kashen district of Xinjiang Uygur Autonomous Region. For Res 2:570-575 (in Chinese)

3. Lantos $G$ (1970) The flexural behavior of steel reinforced laminated timber beams. Wood Sci 2:136-143
4. Bulleit WM, Sandberg LB, Woods GJ (1989) Steel-reinforced glued laminated timber. J Struct Eng 115:433-444

5. Bulleit WM (2007) Reinforcement of wood materials: a review. Wood Fiber Sci 16:391-397

6. Gentile C, Svecova D, Rizkalla SH (2002) Timber beams strengthened with GFRP bars: development and applications. J Compos Constr 6:11-20

7. Borri A, Corradi M, Grazini A (2003) FRP reinforcement of wood elements under bending loads. In: Proceedings of the 10th International Conference on Structural Faults + Repair, London, UK, 1-3 July 2003

8. De la Rosa García P, Escamilla AC, Nieves González García M (2013) Bending reinforcement of timber beams with composite carbon fiber and basalt fiber materials. Compos Part B-Eng 55:528-536

9. Ma SQ, Bunnori NM, Choong KK (2018) Prediction of ultimate Torque of reinforced concrete box beam bonded with CFRP strips. KSCE J Civ Eng 22:4353-4363

10. Plevris N, Triantafillou TC (1992) FRP-reinforced wood as structural material. J Mater Civil Eng 4:300-317

11. Borri A, Corradi M, Grazini A (2005) A method for flexural reinforcement of old wood beams with CFRP materials. Compos Part B-Eng 36:143-153

12. Yang H, Liu W, Shao J, Zhou Z (2008) Study on flexural behavior of timber beams strengthened with FRP". J Build Mater 11:591-597

13. Li YF, Xie YM, Tsai MJ (2009) Enhancement of the flexural performance of retrofitted wood beams using CFRP composite sheets. Constr Build Mater 23:411-422

14. Li YF, Tsai MJ, Wei TF, Wang WC (2014) A study on wood beams strengthened by FRP composite materials. Constr Build Mater 62:118-125

15. Chun Q, Pan JW (2011) Experimental study on bending behavior of timber beams reinforced with CFRP/AFRP hybrid FRP sheets. Adv Mater 255-260:728-732

16. Dhand V, Mittal G, Rhee KY, Park SJ, Hui D (2015) A short review on basalt fiber reinforced polymer composites. Compos Part B-eng 73:166-180

17. Monaldo E, Nerilli F, Vairo G (2019) Basalt-based fiber-reinforced materials and structural applications in civil engineering. Compos Struct 214:246-263

18. De la Rosa García P, Escamilla AC, Nieves González García M (2016) Analysis of the flexural stiffness of timber beams reinforced with carbon and basalt composite materials. Compos Part B-eng 86:152-159

19. GB/T 1938-2009 (2009) Method of testing in tensile strength parallel to grain of wood. China National Standard, Beijing

20. GB/T 1935-2009 (2009) Method of testing in compressive strength parallel to grain of wood. China National Standard, Beijing

21. GB/T 1939-2009 (2009) Method of testing in compression perpendicular to grain of wood. China National Standard, Beijing

22. GB/T 50329-2012 (2012) Standard for test methods of timber structures. China National Standard, Beijing

23. Kim YJ, Harries KA (2010) Modeling of timber beams strengthened with various CFRP composites. Eng Struct 32:3225-3234

24. ANSYS (2009) ANSYS online manual. ANSYS Inc, Canonsburg

25. Lei C, Weidong W, Haitao C (2012) Generalization of Hill's yield criterion to tension-compression asymmetry materials. Sci China Technol Sc 56:89-97

26. Plunkett B, Cazacu O, Barlat F (2008) Orthotropic yield criteria for description of the anisotropy in tension and compression of sheet metals. Int J Plast 24:847-866

27. Bodig J, Jayne BA (1982) Mechanics of wood and wood composite. Van Nostrand Reinhold Company Inc, New York

28. Liang Z, Liu Q, Li X, Liu Y (2014) Paste the CFRP strengthened beams from Xinjiang poplar experimental research and finite element analysis. Sichuan Build Sci 40:95-100 (in Chinese)

29. Bakoss S, Greenland A, Crews K (1999) Bridge deck and industrial heavyduty flooring system based on laminated veneer lumber beams reinforced with carbon fiber composites. Proceedings of the 8th International Conference on Structural Faults + Repair, London, UK, 4-6 July 1999

30. Banjara NK, Ramanjaneyulu K (2017) Experimental and numerical investigations on the performance evaluation of shear deficient and GFRP strengthened reinforced concrete beams. Constr Build Mater 137:520-534

\section{Publisher's Note}

Springer Nature remains neutral with regard to jurisdictional claims in published maps and institutional affiliations. 